\title{
A SERS-based lateral flow assay biosensor for highly sensitive detection of HIV-1 DNA
}

\author{
Xiuli Fu ${ }^{\mathrm{a}}$, Ziyi Cheng ${ }^{\mathrm{a}}$, Jimin $\mathrm{Yu}^{\mathrm{a}}$, Priscilla Choo ${ }^{\mathrm{b}}$, Lingxin Chen ${ }^{\mathrm{c}}$, Jaebum Choo ${ }^{\mathrm{a}, *}$ \\ a Department of Bionano Technology, Hanyang University, Ansan 426-791, South Korea \\ ${ }^{\mathrm{b}}$ Department of Chemistry, Indiana University, Bloomington, IN 47405-102, USA \\ ${ }^{\mathrm{c}}$ Key Laboratory of Coastal Environmental Processes and Ecological Remediation, Yantai Institute of Coastal Zone Research, Chinese Academy of Sciences, \\ Yantai 264003, China
}

\section{A R T I C L E I N F O}

\section{Article history:}

Received 17 August 2015

Received in revised form

29 November 2015

Accepted 30 November 2015

Available online 2 December 2015

Keywords:

Human immunodeficiency virus type 1

(HIV-1)

Surface-enhanced Raman scattering

Gold nanoparticles

DNA

Lateral flow assay

\begin{abstract}
A B S T R A C T
User-friendly lateral flow (LF) strips have been extensively used for point-of-care (POC) self-diagnostics, but they have some limitations in their detection sensitivity and quantitative analysis because they only identify the high cut-off value of a biomarker by utilizing color changes that are detected with the naked eye. To resolve these problems associated with LF strips, we developed a novel surface-enhanced Raman scattering (SERS)-based LF assay for the quantitative analysis of a specific biomarker in the low concentration range. Herein, human immunodeficiency virus type 1 (HIV-1) DNA was chosen as the specific biomarker. Raman reporter-labeled gold nanoparticles (AuNPs) were employed as SERS nano tags for targeting and detecting the HIV-1 DNA marker, as opposed to using bare AuNPs in LF strips. It was possible to quantitatively analyze HIV-1 DNA with high sensitivity by monitoring the characteristic Raman peak intensity of the DNA-conjugated AuNPs. Under optimized conditions, the detection limit of our SERS-based lateral flow assay was $0.24 \mathrm{pg} / \mathrm{mL}$, which was at least 1000 times more sensitive compared to colorimetric or fluorescent detection methods. These results demonstrate the potential feasibility of the proposed SERS-based lateral flow assay to quantitatively detect a broad range of genetic diseases with high sensitivity.
\end{abstract}

(c) 2015 Elsevier B.V. All rights reserved.

\section{Introduction}

Human immunodeficiency virus type 1 (HIV-1) is a retrovirus that attacks the body's immune system making it unable to resist infection. This HIV-1 virus sometimes causes acquired immunodeficiency syndrome (AIDS), which produces disorders and infections and usually leads to death (Douek et al., 2009; Weiss, 1993). Early diagnosis and treatment programs of HIV-1 that are rapid and simple can greatly reduce human mortality rates (Boyle et al., 2013). To date, the enzyme-linked immunosorbent assay (ELISA) test, combined with the confirmatory immunofluorescence Western blot assay, is regarded as the gold standard method for diagnosing HIV-1. When the ELISA test is combined with the Western blot, the rate of false positives has been proven to be extremely low, and the diagnostic accuracy is very high (New York Department of Health, 2011). However, at the point of care (POC) level, this test cannot be reliably performed due to time-consuming sample pretreatments, long assay times and high experimental costs (Lequin, 2005; Zhang et al., 2014). These issues

\footnotetext{
* Corresponding author.

E-mail address: jbchoo@hanyang.ac.kr (J. Choo).
}

restrict further practical applications of standard method in the field, raising the need for facile, direct, rapid and sensitive approach in accurate detection of HIV-1 virus.

The DNA polymerase chain reaction (PCR) is another sensitive detection technique for HIV-1 (Kuritzkes et al., 2003; Nkeze et al., 2014). This offers high accuracy and sensitivity but is poorly suited for implementation in low-resource settings due to the complicated sample pretreatment, the need for trained technicians, and the three to four week turnaround time (Crannell et al., 2014; Fang et al., 2014; Gao et al., 2014; Rohrman and Richards-Kortum, 2012). Furthermore, false-negative results may occur because the PCR involves a thermo-cyclic amplification process that utilizes minute quantities of DNA.

A paper-based lateral flow (LF) strip, which combines nanoparticles with conventional chromatographic separation, has attracted significant interest because nanoparticles are favorable for signal amplification, which is used to achieve high sensitivity and selectivity for target analysis (He et al., 2010; Mao et al., 2009a; Xu et al., 2009). LF strips are considered to be one of the most promising techniques due to their simplicity, rapid analysis, relatively low interference due to chromatographic separation, low costs, long-term stability over a wide range of climates, and their ability to be easily used with no requirements for skilled technicians 
(Chen et al., 2013; Mao et al., 2009b; Shen et al., 2013). Until now, several different types of LF strip have been developed to detect nucleic acids including DNA or miRNA (Fang et al., 2014; Gao et al., 2014; He et al., 2010; Hu et al., 2013; Mao et al., 2009b). For example, a dry-reagent strip biosensor based on a molecular beaconfunctionalized gold nanoparticle probe has been developed for rapid and quantitative detection of specific nucleic acids (Mao et al., 2009b). A simple one-step signal amplification strategy, using oligonucleotide-linked gold nanoparticle aggregates, has also been developed to enhance the sensitivity of the nucleic acids LF assay (Hu et al., 2013). Nonetheless, some of the problems associated with these analytical methods, including low sensitivity and limits in quantitative analysis, have made these LF biosensors less attractive. In order to resolve these problems, optical reading systems should be employed. Up to date, phase contrast and fluorescence readers have commonly been used to measure color intensities produced in a test. However, these optical readers will suffer from lack of precision and sensitivity (Li et al., 2010).

Surface-enhanced Raman scattering (SERS) spectroscopy is a promising analytical technique that can be used to overcome the aforementioned challenges due to its high sensitivity. When reporter molecules are adsorbed onto the nanoparticle surface, their SERS signals are greatly increased at SERS-active sites well known as "hot spots" when exposed to excitation light source due to electromagnetic and chemical enhancement effects. Using this technique, the detection sensitivity can be increased up to 10-14 orders of magnitude higher than that of conventional Raman spectroscopy (Kneipp et al., 1999; Nie and Emory, 1997; Wang et al., 2013a, 2013b; Yoon et al., 2013). This enhancement has shown promises in overcoming low sensitivity problems inherent in Raman and fluorescence spectroscopy. Gold nanoparticles (AuNPs) are most widely used as optical enhancing agent in SERS due to their high SERS enhancement effect through homogeneous aggregation (Lee and Meisel, 1982; Wang et al., 2013a, 2013b), easy preparation and modification, and excellent biocompatibility with biomolecules such as proteins (Chapman et al., 2015; Wu et al., 2013, 2015), antibodies (Chen et al., 2008; Song et al., 2014; Wang et al., 2013a, 2013b), and DNAs (Kim et al., 2011; Sun et al., 2011).

Herein, we propose a novel SERS-based LF assay for the quantitative analysis of HIV-1 DNA. In this system, oligonucleotides (detection DNA probes) and Raman reporter (malachite green isothiocyanate (MGITC))-functionalized AuNPs were used as SERS nano tags. MGITC was used as Raman reporter molecule due to its strong Raman enhancement effect. These MGITC-AuNPs-DNA SERS tags were used as an alternative to the oligonucleotide-labeled AuNPs that are utilized in conventional LF strips. Quantitative detection can be performed by recording the Raman intensity change on the test line. The experimental conditions, including the types of running buffer, the amount of BSA in the running buffer and the amount of DNA-conjugated AuNPs loaded on the conjugate pad, were optimized for high sensitivity detection of HIV-1 DNA. The assay results were compared to those obtained from a commercially available HIV-1 DNA fluorescence assay kit in order to validate our method's feasibility for clinical application. To the best of our knowledge, this is the first report of a SERS-based HIV1 DNA assay biosensor. This approach provides new insights into the early detection of HIV-1.

\section{Experimental section}

\subsection{Reagents and materials}

Gold (III) chloride trihydrate $\left(\mathrm{HAuCl}_{4}\right)$, trisodium citrate, tris(2carboxyethyl) phosphine hydrochloride (TCEP), streptavidin, $\mathrm{Na}_{3} \mathrm{PO}_{4} \cdot 12 \mathrm{H}_{2} \mathrm{O}$, sucrose, Tween 20 , sodium chloride--sodium citrate (SSC) buffer solution ( $\mathrm{pH} 7.0$ ), sodium dodecyl sulfate (SDS), bovine serum albumin (BSA), and Tris- $\mathrm{HCl}$ buffer solution were purchased from Sigma-Aldrich (St. Louis, MO, USA). Malachite green isothiocyanate (MGITC), phosphate buffer saline (PBS, pH 7.4, $0.01 \mathrm{M})$, and the Quant-iT ${ }^{\mathrm{TM}}$ PicoGreen ${ }^{\circledR}$ dsDNA assay kit were purchased from Life Technologies (Seoul, South Korea). The nitrocellulose (NC) membrane attached to a backing card (Hi-flow plus HF180) was purchased from the Millipore Corporation (Billerica. MA, USA). Absorbent pads (CF4) were purchased from Whatman-GE Healthcare (Pittsburgh, PA, USA).

The target HIV-1 DNA and oligonucleotide probes used in this study were purchased from Integrated DNA Technologies, Inc. (Coralville, IA, USA). They have the following sequences (Hu et al., 2013):

Target DNA: 5'-AGT AGT GTG TGC CCG TCT GTT GTG TGA CTC TGG TAA CTA GAG ATC CCT CAG AC-3'

Detection DNA: 5'-SH-( $\left.\mathrm{CH}_{2}\right)_{6}$-TTTTTTTTTT GT CTG AGG GAT CTC TAG TTA-3'

Capture DNA: 5'-ACA GAC GGG CAC ACA CTA CT-biotin-3'

Control DNA: 5'-biotin-TAA CTA GAG ATC CCT CAG AC-3'

Non-complementary DNA: 5'-GCC TCA ATA AAG CTT GCC TTG AGT GCT TGT GGA AAA TCT CTA GCA GTG GCG CC-3'

All other chemicals were of analytical reagent grade and used without further purification. All solutions were prepared with ultrapure ( $>18.2 \mathrm{M} \Omega$ ) water from a Millipore Milli-Q water purification system (Millipore Corporation, Billerica, MA, USA).

\subsection{Instrumentation}

The DCI 302 dispenser (ZETA Corporation, Seoul, South Korea) was used to immobilize, capture, and control DNAs on the test and control lines of the NC membrane. A programmable paper cutter (GCI-800, ZETA Corporation, Seoul, South Korea) was used to prepare strips of a desired size. High-magnification transmission electron microscopy (TEM) images were obtained with a JEOL JEM $2100 \mathrm{~F}$ instrument (JEOL, Tokyo, Japan) at an accelerating voltage of $200 \mathrm{kV}$. To obtain TEM images of DNA-conjugated AuNPs, a small drop of colloidal dispersion of nanoparticles was deposited onto a piece of copper grid and dried in room temperature under atmospheric pressure. Scanning electron microscopy (SEM) images were acquired using a MIRA3 (TESCAN, Brno, Czech Republic) instrument at an accelerating voltage of $20 \mathrm{kV}$. To obtain SEM images of LF strips, the nanoparticle dispersions were diluted with water and then samples were dried under ambient conditions. Dynamic light scattering (DLS) data were collected using a Nano ZS-90 apparatus (Malvern Instruments, Malvern, UK). A Cary 100 spectrophotometer (Varian, Salt Lake City, UT, USA) was used to acquire UV-visible absorption spectra. All Raman measurements were carried out using an inVia Renishaw Raman microscope system (Renishaw, New Mills, UK).

\subsection{Preparation of gold nanoparticles (AuNPs)}

AuNPs were prepared according to the citrate-reduction method developed by Frens (1973). All glassware used in this preparation was thoroughly cleaned in aqua regia $\left(\mathrm{HCl} / \mathrm{HNO}_{3}\right.$, volume ratio 3:1), rinsed with doubly distilled water, and dried in an oven prior to use. Typically, $50 \mathrm{~mL}$ of a $0.01 \% \mathrm{HAuCl}_{4}$ solution was boiled thoroughly. Then, $0.5 \mathrm{~mL}$ of a $1 \%$ trisodium citrate solution was added rapidly under vigorous stirring. The solution was boiled for another $15 \mathrm{~min}$, during which time its color changed from pale yellow to brilliant red. After cooling to room temperature while stirring, the colloids were stored at $4{ }^{\circ} \mathrm{C}$ for further use. The final concentration of the AuNPs solution was estimated to be $0.12 \mathrm{nM}$. UV-vis spectroscopy and TEM were used to identify the average size of AuNPs (Fig. S1). The average diameter of AuNPs was estimated to be $30-40 \mathrm{~nm}$. 
(A) Step 1: Activation

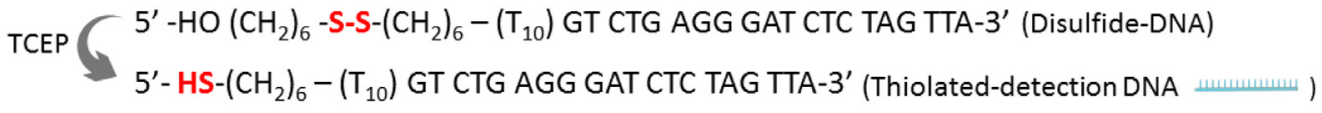

Step 2: Conjugation

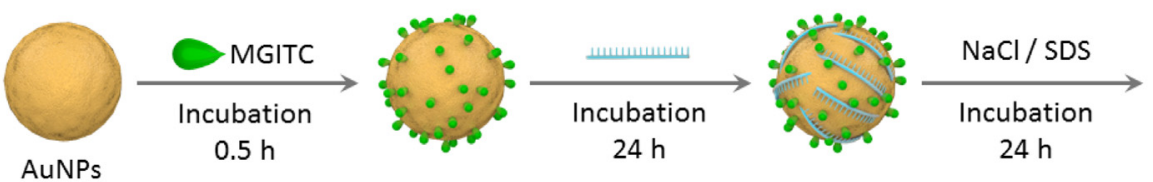

(B)

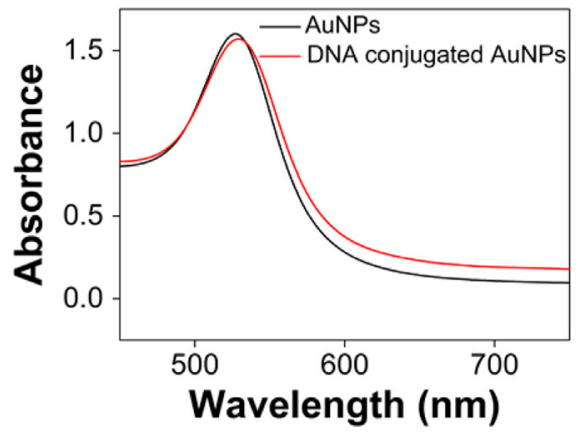

(C)

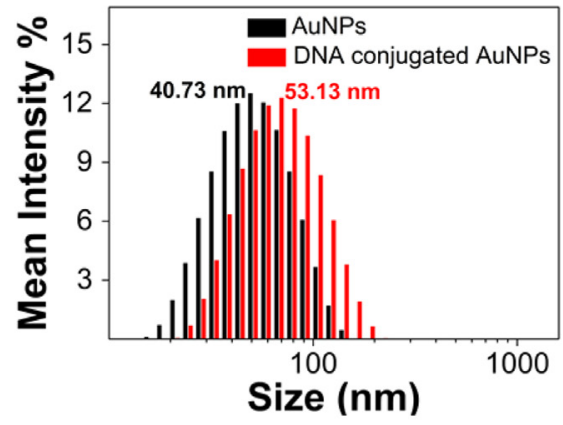

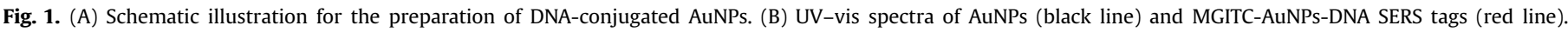
(C) Dynamic light scattering data for the size distributions of AuNPs (black column) and DNA-conjugated AuNPs (red column).

\subsection{Preparation of DNA-conjugated AuNPs}

To prepare a Raman active probe, Raman reporter MGITC molecules were adsorbed onto the surface of AuNPs (Fig. 1A) (Yoon et al., 2013). First, $0.5 \mu \mathrm{L}$ of a $10^{-4} \mathrm{M}$ MGITC solution was added to $1.0 \mathrm{~mL}$ of the AuNPs solution. This mixture was reacted for $0.5 \mathrm{~h}$ under stirring. Then, thiolated DNAs were conjugated on the surface of MGITC-functionalized AuNPs (Yoon et al., 2013). Before the conjugation, thiolated DNAs were activated as follows: $100 \mu \mathrm{L}$ of thiolated DNA in the disulfide form was mixed with $100 \mu \mathrm{L}$ of $5 \mathrm{mM}$ TCEP in $100 \mathrm{mM}$ Tris- $\mathrm{HCl}$ (pH 7.4). The mixture was incubated for $1 \mathrm{~h}$ at room temperature to produce free thiol groups via the reduction of disulfide groups. Conjugation reactions were carried out by adding activated DNA probes to $1 \mathrm{~mL}$ of the functionalized AuNP solution. This mixture was maintained at room temperature for $24 \mathrm{~h}$. Subsequently, a $2 \mathrm{M} \mathrm{NaCl}$ solution containing $1 \%$ SDS was added and reacted for another $24 \mathrm{~h}$. Then, the mixture was centrifuged at $7000 \mathrm{rpm}$ for $10 \mathrm{~min}$ to remove excess reagents. After discarding the supernatant, the remaining red pellets were washed, recentrifuged, and redispersed in $50 \mu \mathrm{L}$ of an aqueous solution containing $20 \mathrm{mM} \mathrm{Na}_{3} \mathrm{PO}_{4}, 5 \% \mathrm{BSA}, 0.25 \%$ Tween 20 , and $10 \%$ sucrose.

\subsection{Preparation of the DNA probe-immobilized lateral flow strip}

$2 \mathrm{mg} / \mathrm{mL}$ streptavidin was dissolved in the $0.01 \mathrm{M}$ PBS solution ( $\mathrm{pH}$ 7.4). Then, $250 \mu \mathrm{L}$ of the streptavidin solution and $250 \mu \mathrm{L}$ of the biotinylated DNA probe solution were mixed and reacted for $1 \mathrm{~h}$ at room temperature. The resulting conjugates were purified by centrifugation at $6000 \mathrm{rpm}$ with a centrifugal filter (cutoff 30000 , Millipore) for $30 \mathrm{~min}$. These conjugates were dissolved in a PBS solution for further use.

The SERS lateral flow strip consisted of a sample pad, a conjugate pad, an NC membrane, and an absorbent pad. Each component was assembled on a plastic adhesive backing layer, and both ends of the component were overlapped ( $2 \mathrm{~mm}$ length) in sequence to ensure solution migration as shown in Scheme 1A.
The plate was then cut into 4-mm-wide strips using a paper cutter. The test and control lines were separately prepared by dispensing the streptavidin-biotinylated capture probe and the streptavidinbiotinylated control probe solution. The strips with lines were dried at $37^{\circ} \mathrm{C}$ for $1 \mathrm{~h}$. The DNA-conjugated SERS nano tag $(4 \mu \mathrm{L})$ was added to the conjugate pad and dried at room temperature for $10 \mathrm{~min}$. The integrated strips were stored at $4{ }^{\circ} \mathrm{C}$ in a dry state for detection. $150 \mu \mathrm{L}$ aliquots of the standard target solutions in the running buffer $(4 \times$ SSC buffer containing $1 \%$ BSA) were applied to the sample pad. For quantitative measurement, the Raman intensity of the test line was collected and analyzed by the Raman instrument.

\section{Results and discussion}

\subsection{Principle of the SERS-based lateral flow DNA assay}

The operating principle of the SERS-based LF assay for HIV-1 DNA is illustrated in Scheme 1. This assay is based on the sandwich-type "DNA-conjugated AuNPs-target DNA-capture DNA" hybridization reactions. The DNA-conjugated AuNPs were dispensed on the conjugate pad, which was designed to bind specifically with the target DNA (Scheme 1A). Streptavidin-biotinylated capture DNA (complementary, with a part of the target DNA) and streptavidin-biotinylated control DNA (complementary, with a detection DNA probe on the MGITC-AuNPs surface) were pre-immobilized on the NC membrane to form the test and control lines, respectively (Scheme 1A). Typically, the sample solution containing the desired amount of target DNA was applied onto the sample pad. Subsequently, the solution migrated by capillary action toward the absorption pad. When the migrating solution passed through the conjugate pad, the target DNA became hybridized with the detection DNA that was immobilized on AuNPs to form the complex and continued to migrate along the strip. As the resulting complexes reached the test line, they were captured by the probe DNA that was immobilized on the test line. This capture 
(A)

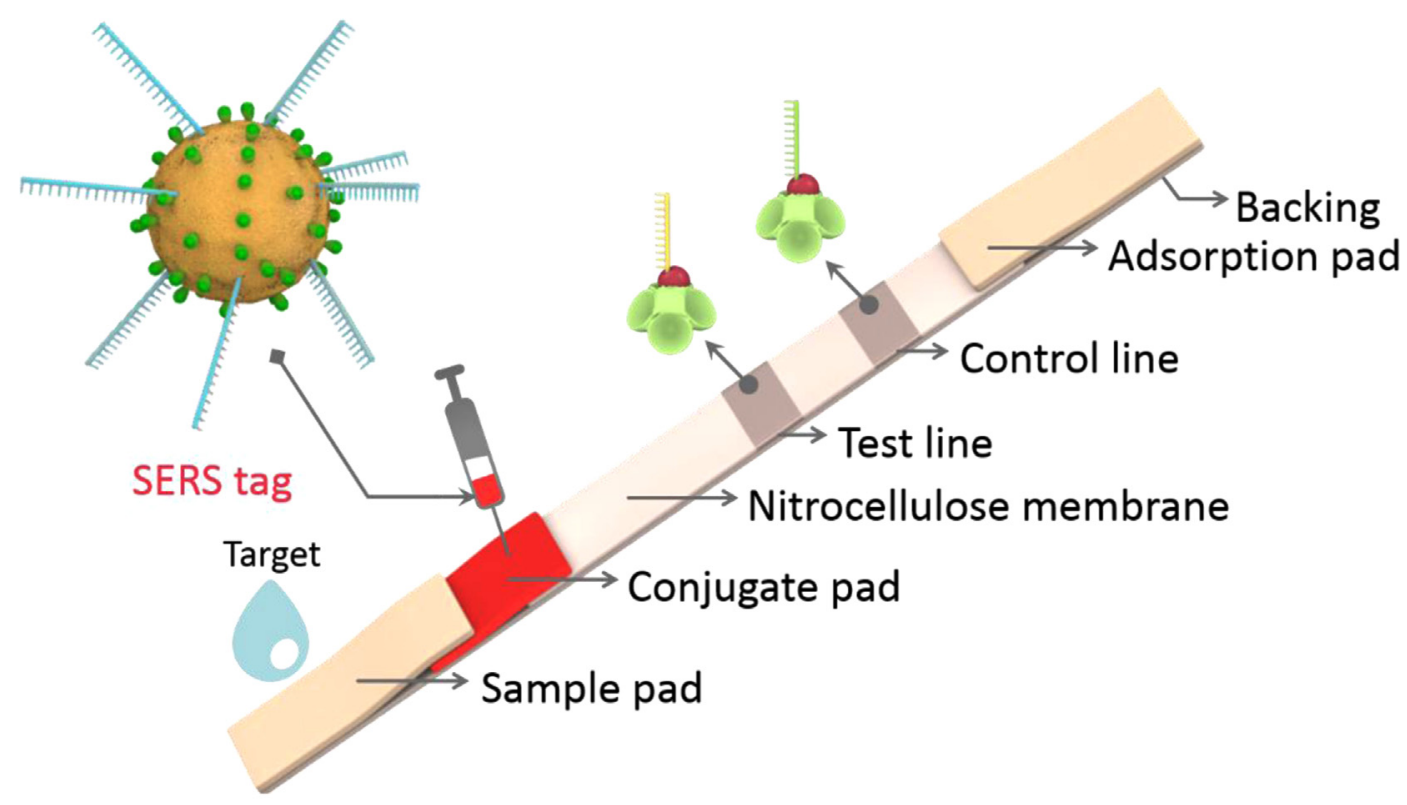

(B)

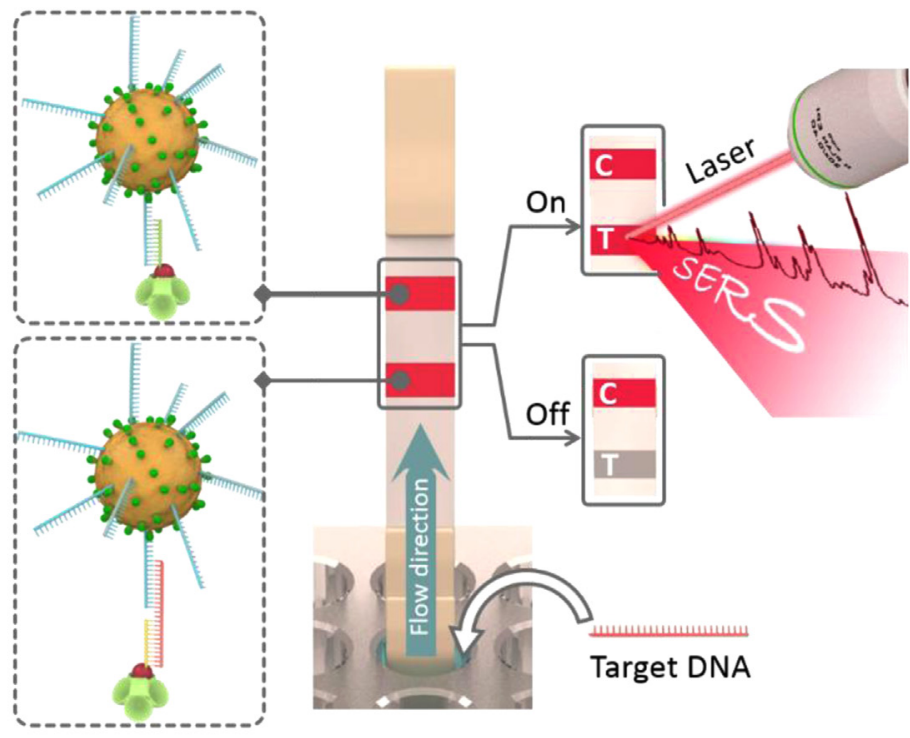

Target DNA:

5'-AGT AGT GTG TGC CCG TCT GTT GTG TGA CTC TGG TAA

CTA GAG ATC CCT CAG AC-3'

\section{Thiolated-detection DNA:}

5' - SH- $\left(\mathrm{CH}_{2}\right)_{6}$-TTTTTTTTTT GT CTG AGG GAT CTC TAG TTA- $3^{\prime}$

Biotinylated-capture DNA:

5'-ACA GAC GGG CAC ACA CTA CT-biotin-3'

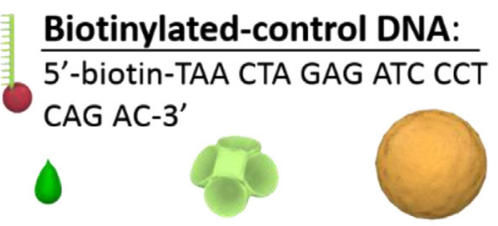

MGITC Streptavidin AuNPs

Scheme 1. (A) Schematic illustration of the configuration and (B) the measurement principle of the SERS-based lateral flow assay for quantification of HIV-1 DNA. (C is the control line and $\mathrm{T}$ is the test line).

occurred due to the second hybridization between the target DNA and the capture DNA, resulting in the formation of sandwich complexes. Then, the excess DNA-conjugated AuNPs continued to migrate through the control line and were captured by the probe DNA that was pre-immobilized on the control line. In this case, two red bands were shown (Scheme 1B, “on"). In the absence of any target DNA, only one red band was observed on the control line; this shows that the biosensor was working properly (Scheme 1B, “off"). Quantitative analysis was performed by reading the Raman intensity of the red band on the test line by using the Raman instrument.

\subsection{Quantitative analysis of HIV-1 DNA using the SERS-based lateral flow assay}

To confirm whether or not the detection DNA was efficiently bound onto the surface of AuNPs, the UV-vis and DLS spectra for DNA-conjugated AuNPs were measured and analyzed. The UV-vis absorption band for DNA-conjugated AuNPs displays a small red shift compared to that of AuNPs, as shown in Fig. 1B. In addition, the DLS distribution demonstrates that the diameter of DNAconjugated AuNPs is significantly larger than that of AuNPs (Fig. 1C). All these observations demonstrate that DNA was determined to be successfully bound onto the surface of AuNPs.

In the present work, the proof-of-concept of the SERS-based LF platform for the quantitative analysis of the HIV-1 target DNA is introduced. As expected from the original design, the DNA-conjugated AuNPs were captured on the test line by the "DNA-conjugated AuNPs-HIV-1 DNA-capture DNA" hybridization reaction, and the presence of HIV-1 DNAs can be identified through a color change on the test line. At the same time, DNA-conjugated AuNPs were also used as optical detection probes for the quantitative evaluation of target DNAs. When HIV-1 DNAs are present in a sample, a cluster of DNA-conjugated AuNPs are observed on the test line; this observation is responsible for the color change to red ("on", Fig. 2). In addition, it is also possible to achieve a 


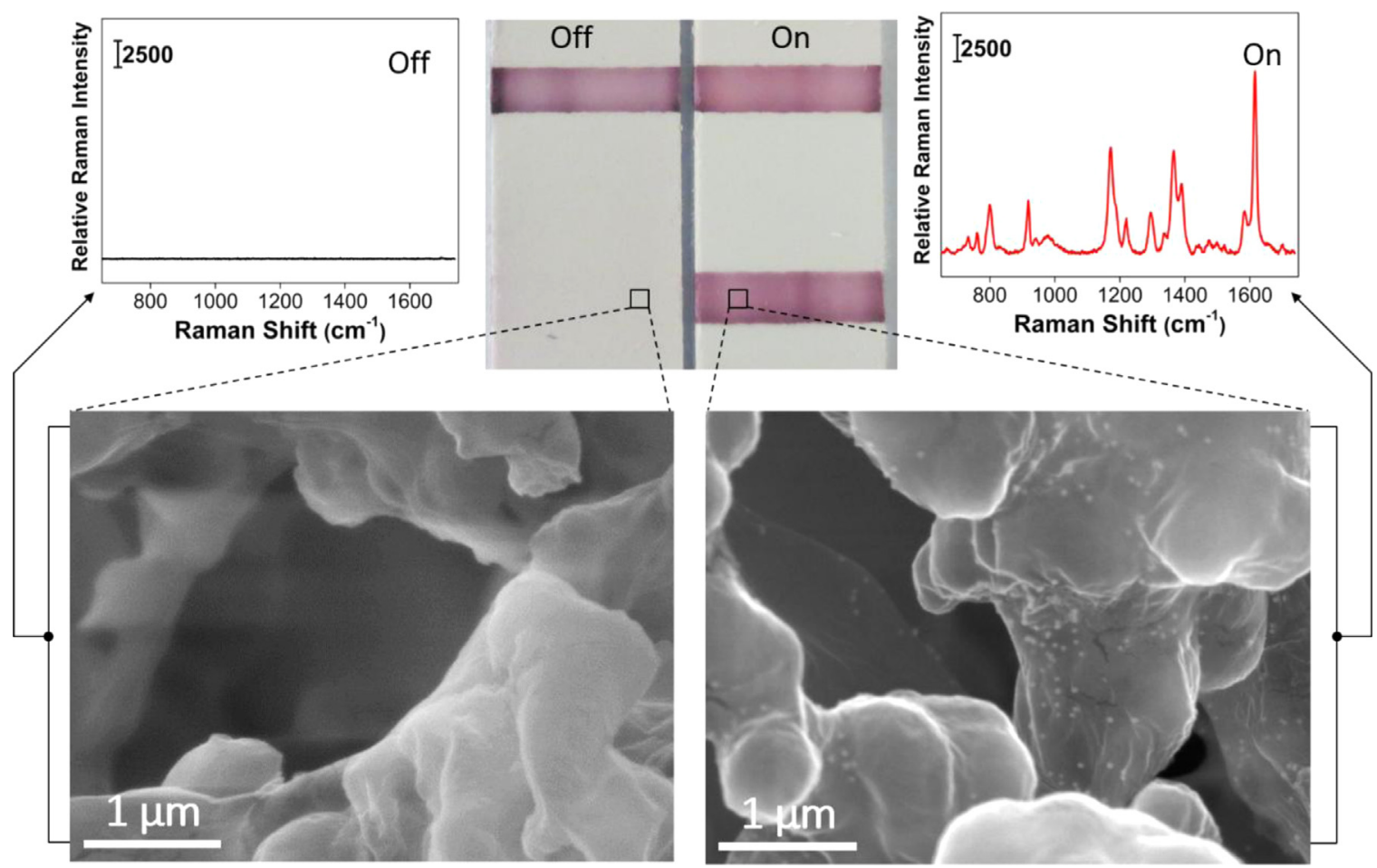

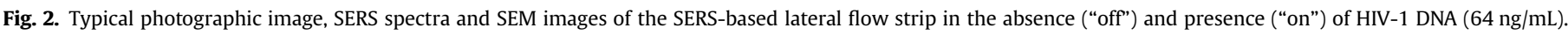

quantitative result by measuring the SERS signal intensity. In the absence of the target DNA, however, no color change was observed ("off", Fig. 2) because no DNA hybridization occurs on the test line. In this case, no SERS signal was observed on the test line. Consequently, the presence of HIV-1 DNA could be identified by the naked eye. Additionally, quantitative analysis of HIV-1 DNA was achieved by monitoring the characteristic SERS peak intensity of the DNA-conjugated AuNPs.

\subsection{Optimization of the SERS lateral flow HIV-1 DNA assay parameters}

To optimize the various conditions for the hybridization of HIV1 DNA and the SERS detection, various factors, including the running buffer, the amount of BSA in the running buffer, and the amount of DNA-conjugated AuNPs loaded on the conjugate pad, have been systematically investigated.

\subsubsection{Effect of running buffer solution}

The running buffer solution strongly affects the performance of the sensor and the hybridization efficiency of the "DNA-conjugated AuNPs-HIV-1 DNA-capture DNA" reaction. Three different types of running buffer solutions, including Tris-HCl, PBS, and SSC, were tested. As shown in Fig. S2A, the Raman intensity for the SSC running buffer is significantly higher than that for the Tris- $\mathrm{HCl}$ or PBS buffer solutions. Thus, the SSC buffer solution was used in this work. The concentration of the SSC buffer is another important issue that must be considered. In this regard, different concentrations of the SSC buffer (ranging from $2 \mathrm{x}$ to $8 \mathrm{x}$ ) were tested on the sample pad (Fig. S2B). On the basis of our experimental results, the $4 \mathrm{x}$ SSC buffer was estimated to be the optimum concentration for the SERS-based HIV-1 DNA assay.

The amount of BSA in the running buffer can also influence the sensitivity of the SERS-based HIV-1 LF assay. Fig. S2C displays variations in the SERS intensity for the $16 \mathrm{ng} / \mathrm{mL}$ target HIV-1 DNA with different concentrations of BSA in the SSC running buffer. As shown in this figure, the Raman intensity gradually decreases as the BSA concentration is increased. Thus, a BSA concentration of $1.0 \%$ was selected for the following experiments.

\subsubsection{Effect of DNA-conjugated AuNPs}

Optimizing the concentration of the DNA-conjugated AuNPs is also important for the quantitative analysis of the target HIV-1 DNA. This is the case because the Raman intensity of the test line depends on the number of DNA-conjugated AuNPs inside the laser beam. The concentration effect of the DNA-conjugated AuNPs was investigated by dispensing different volumes of AuNPs onto the conjugation pad. Fig. S2D shows the Raman signal intensity variation as a function of the volume of the DNA-conjugated AuNPs (from $1 \mu \mathrm{L}$ to $4 \mu \mathrm{L}$ ). Here, a concentration of $4 \mu \mathrm{L}$ was estimated to be the optimum volume for the DNA-conjugated AuNPs.

\subsection{Analytical performance of the SERS-based lateral flow biosensor}

Under the optimized experimental conditions, the analytical performance of the SERS lateral flow assay was examined with different concentrations of the target DNA. Fig. 3A shows the photo image of the SERS-based lateral flow biosensor in the presence of various target DNA concentrations ranging from 0 to $64 \mathrm{ng} / \mathrm{mL}$. As the HIV-1 DNA concentration increased, more sandwich complexes are formed, and the red color intensity becomes darker. This also means that more DNA-conjugated AuNPs are captured on the test line, and the SERS intensity gradually increases as the concentration of the HIV-1 DNA increases (Fig. 4A). The red band could be observed with the naked eye at concentrations as low as $80 \mathrm{pg} / \mathrm{mL}$. Quantitative analysis of HIV-1 DNA was also performed by recording the Raman intensity of the test line. For each concentration, the SERS signals for five different points on the test line were averaged to obtain a reproducible intensity value (Fig. 3A). Fig. S4 displays the intensity variation of Raman peak at $1614 \mathrm{~cm}^{-1}$ of five different spots for six different HIV1 DNA concentrations, and their small intensity variations indicate that the SERS signals were achieved with a good analytical reproducibility. Fig. 3B displays the SERS spectra for concentrations ranging from 0 to $800 \mathrm{pg} / \mathrm{mL}$. 

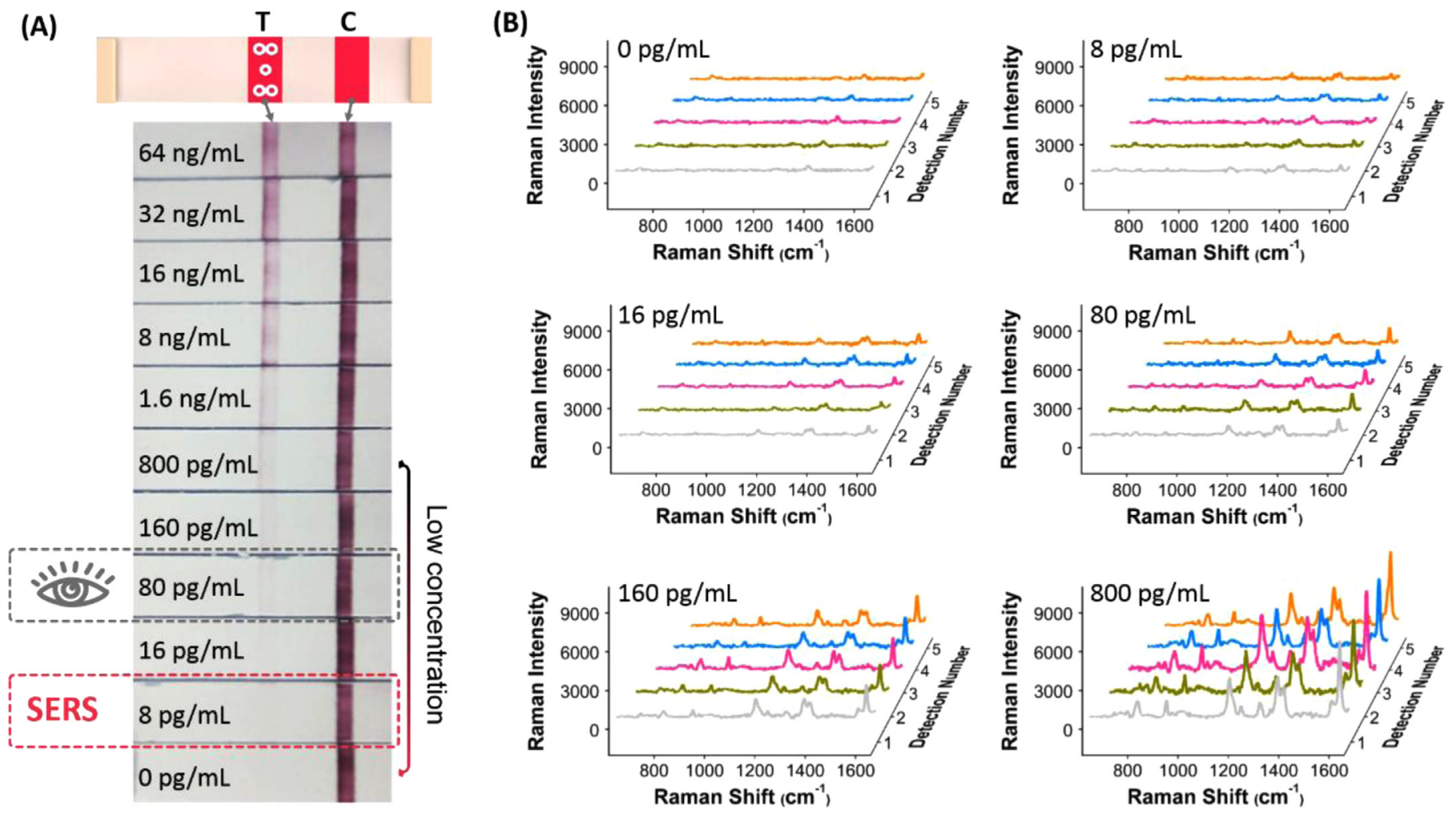

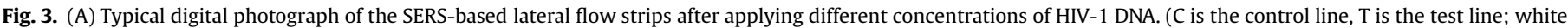
circles show the detection point) (B) SERS spectra for lower concentrations of HIV-1 DNA from 0 to $800 \mathrm{pg} / \mathrm{mL}$. Five tests were performed at each concentration.

Fig. 4 shows the SERS spectra (Fig. 4A) for eleven different concentrations of HIV-1 DNA and their corresponding calibration curves (Fig. 4B). Fig. S5 shows the SEM images of the sandwich complexes for six different concentrations of HIV-1 DNA $(0 \mathrm{pg} / \mathrm{mL}$, $16 \mathrm{pg} / \mathrm{mL}, 160 \mathrm{pg} / \mathrm{mL}, 1600 \mathrm{pg} / \mathrm{mL}, 16 \mathrm{ng} / \mathrm{mL}$, and $32 \mathrm{ng} / \mathrm{mL})$. As shown in this figure, more DNA-conjugated AuNPs are captured on the test line through the sandwich-type DNA hybridization reactions at increased HIV-1 DNA concentrations. However, no DNAconjugated AuNPs are attached on the test line in the absence of HIV-1 DNA. These SEM images provide strong evidence of the increased formation of sandwich-type complexes as the target DNA concentration is increased. The resulting calibration plot of the Raman intensity versus the target HIV-1 DNA concentration is displayed in Fig. 4B. In this figure, the sigmoidal curve (Fig. 4B left) was converted to a linear curve via log-log transformation (Fig. 4B right), which is able to be used for the determination of HIV-1 DNA concentration from an unknown sample. The limit of detection (LOD) value was determined by the IUPAC standard method $\left(\mathrm{LOD}=y_{\text {blank }}+3 \times \mathrm{SD}_{\text {blank }}, y_{\text {blank }}\right.$ is the average signal intensity at zero and $S_{\text {blank }}$ is the standard deviation of the blank measurements.). On the basis of this equation, the LOD was determined to be $\sim 0.24 \mathrm{pg} / \mathrm{mL}$ (Cho et al., 2006; Zhao et al., 2014). This value is at least 1000 times more sensitive than the values that were previously reported by the color intensity approach (Hu et al., 2013; Mao et al., 2009b) or by the fluorescent detection method (Guo et al., 2014). The improvement of the detection sensitivity was attributed to the significant enhancement provided by the DNAconjugated AuNPs.

The binding specificity of the SERS-based LF assay was also evaluated. As shown in Fig. S6, in the presence of $64 \mathrm{ng} / \mathrm{mL}$ noncomplementary DNA (5'-GCC TCA ATA AAG CTT GCC TTG AGT GCT TGT GGA AAA TCT CTA GCA GTG GCG CC-3'), the Raman intensity on the test line is almost the same as the intensity of the blank. Alternatively, with the same concentration of the target HIV-1 DNA (5'-AGT AGT GTG TGC CCG TCT GTT GTG TGA CTC TGG TAA
CTA GAG ATC CCT CAG AC-3'), a remarkable Raman intensity increase is observed. These results demonstrate that the SERS-based LF assay only responds to the target DNA and that it has a strong specificity toward the target DNA.

\subsection{Performance comparison between the SERS-based lateral flow assay and the commercially available assay kit}

To investigate the potential clinical application of our SERS-based LF assay, we compared its analytical performance with that of a commercially available HIV-1 assay kit (Quant-iTrм PicoGreen ${ }^{\circledR}$ ). Eight different concentrations of HIV-1 DNA, ranging from 8 to $8000 \mathrm{pg} / \mathrm{mL}$, were prepared and tested. The assay using the commercial kit was performed according to the provided protocol (Fig. S7). As shown in Fig. 5, the minimum detectable concentration of HIV-1 DNA using the commercial assay kit is $80 \mathrm{pg} / \mathrm{mL}$; this is much higher than the minimum detection limit measured by our SERSbased lateral flow assay $(8 \mathrm{pg} / \mathrm{mL})$. Furthermore, it is also possible to carry out a highly sensitive quantitative assay of HIV-1 DNA in the lower concentration range ( $>100 \mathrm{pg} / \mathrm{mL}$ ). This means that our SERSbased LF assay is suitable for the early diagnosis of HIV-1 infections in the clinical laboratory.

\section{Conclusion}

User-friendly LF strips are considered to be attractive POCbased diagnostic tools due to their simple and rapid self-diagnostic characteristics. However, their application has been limited to diagnostics that utilize high concentration biomarkers (at $\mathrm{mg} / \mathrm{mL}$ or $\mu \mathrm{g} / \mathrm{mL}$ levels) in blood or urine; these types of tests include analyzing glucose for diabetic tests and human chorionic gonadotropin (HCG) for pregnancy tests. Furthermore, quantitative analysis with POC-based LF strips is difficult because they rely on the naked eye to identify changes in color. 
(A)
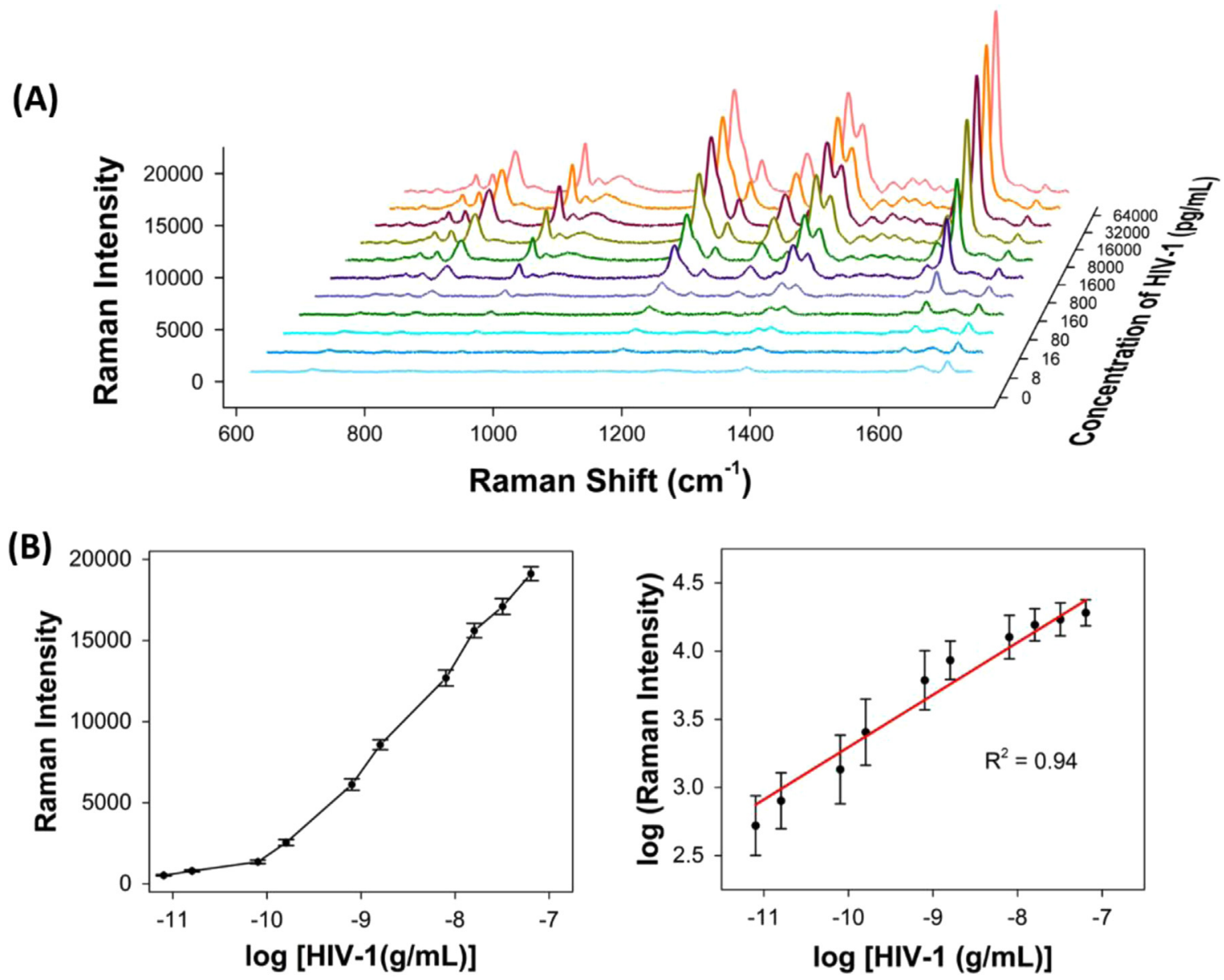

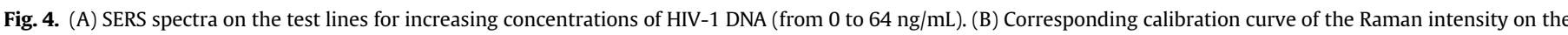

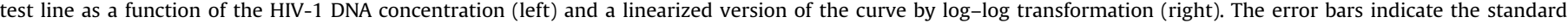
deviations calculated from five parallel measurements. Assay time: $15 \mathrm{~min}$.

To resolve these problems, we have successfully developed a novel SERS-based DNA LF biosensor for the simple, rapid, and sensitive detection of a specific biomarker. Quantitative analysis in the low concentration range is also possible using a Raman instrument reader. Herein, Raman reporter-labeled AuNPs were used as SERS detection probes (as opposed to bare AuNPs, which are used in POC-based LF strips). It was possible to quantify the amount of the model target HIV-1 DNA marker with high reproducibility. Under optimal conditions, a linear relationship between the Raman intensity and the HIV-1 DNA concentration was obtained in the $8 \mathrm{pg} / \mathrm{mL}$ to $64 \mathrm{ng} / \mathrm{mL}$ range with a detection limit of $0.24 \mathrm{pg} / \mathrm{mL}$.

Recently, many commercially available portable Raman systems have been developed. Using the integrated system composed of a portable Raman spectrometer and a LF strip, it is expected that a highly accurate quantitative analysis of target DNA biomarkers can be achieved successfully in the field. The SERS-based immunoassay using integrated portable Raman system is under
(A)

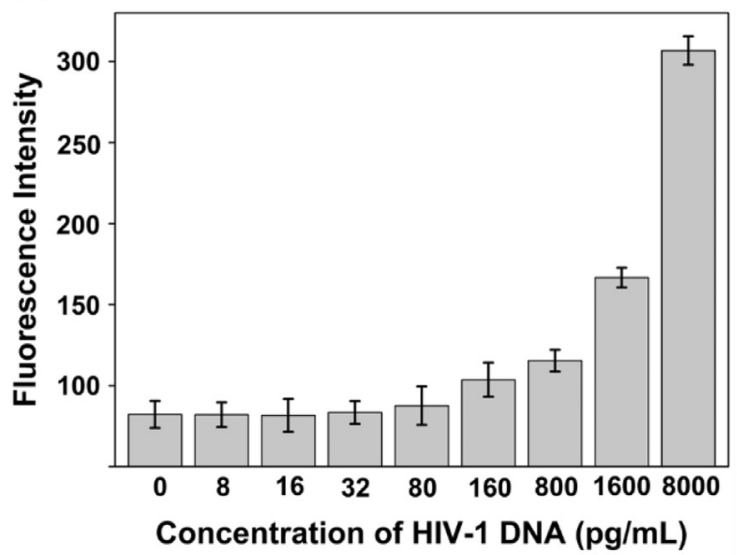

(B)

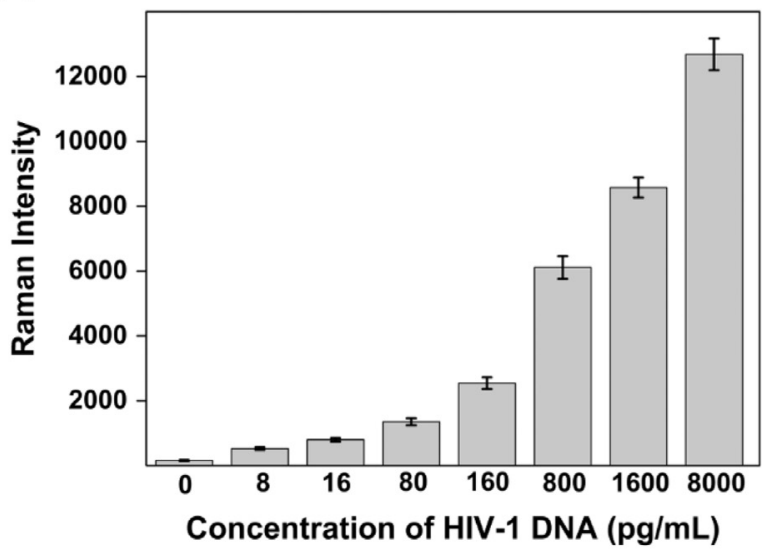

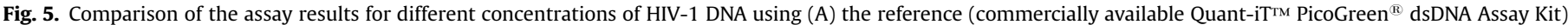
method and (B) the proposed (SERS-based lateral flow assay) method. The error bars indicate the standard deviations calculated from five measurements. 
investigation now. This work takes a step closer to the potential feasibility of SERS-based LF biosensor for application in POC diagnosis. We expect that this novel SERS-based LF biosensor can be used easily and simultaneously to detect multiple DNA biomarkers.

\section{Acknowledgments}

The National Research Foundation of Korea supported this work through Grant numbers 2008-0061891 and 2009-00426. The Nano Material Technology Development Program also supported this work, through the National Research Foundation of Korea, funded by the Ministry of Science, ICT and Future Planning (2012M3A7B4035288).

This work was partially supported by a grant of the National Natural Science Foundation of China through Grant number 21275158.

\section{Appendix A. Supplementary material}

Supplementary data associated with this article can be found in the online version at http://dx.doi.org/10.1016/j.bios.2015.11.099.

\section{References}

Boyle, D.S., Lehman, D.A., Lillis, L., Peterson, D., Singhal, M., Armes, N., Parker, M., Piepenburg, O., Overbaugh, J., 2013. Mbio 4, e00135.

Chapman, R., Lin, Y.Y., Burnapp, M., Bentham, A., Hillier, D., Zabron, A., Khan, S., Tyreman, M., Stevens, M.M., 2015. ACS Nano 9, 2565-2573.

Chen, J.H., Zhou, X.M., Zeng, L.W., 2013. Chem. Commun. 49, 984-986.

Chen, J.W., Lei, Y., Liu, X.J., Jiang, J.H., Shen, G.L., Yu, R.Q., 2008. Anal. Bioanal. Chem. 392, 187-193.

Cho, J.H., Han, S.M., Paek, E.H., Cho, I.H., Paek, S.H., 2006. Anal. Chem. 78, 793-800.

Crannell, Z.A., Castellanos-Gonzalez, A., Irani, A., Rohrman, B., White, A.C., RichardsKortum, R., 2014. Anal. Chem. 86, 2565-2571.

Douek, D.C., Roederer, M., Koup, R.A., 2009. Annu. Rev. Med. 60, 471-484.

Fang, Z.Y., Wu, W., Lu, X.W., Zeng, L.W., 2014. Biosens. Bioelectron. 56, 192-197.

Frens, G., 1973. Nat. Phys. Sci. 241, 20-22.
Gao, X.F., Xu, H., Baloda, M., Gurung, A.S., Xu, L.P., Wang, T., Zhang, X.J., Liu, G.D., 2014. Biosens. Bioelectron. 54, 578-584.

Guo, Q.Q., Chen, Y., Song, Z.P., Guo, L.Q., Fu, F.F., Chen, G.N., 2014. Anal. Chim. Acta 852, 244-249.

He, Y.Q., Zeng, K., Gurung, A.S., Baloda, M., Xu, H., Zhang, X.B., Liu, G.D., 2010. Anal. Chem. 82, 7169-7177.

Hu, J., Wang, L., Li, F., Han, Y.L., Lin, M., Lu, T.J., Xu, F., 2013. Lab Chip 13, 4352-4357. Kim, N.H., Lee, S.J., Moskovits, M., 2011. Adv. Mater. 23, 4152-4156.

Kneipp, K., Kneipp, H., Itzkan, I., Dasari, R.R., Feld, M.S., 1999. Chem. Rev. 99, 2957-2975.

Kuritzkes, D.R., Grant, R.M., Feorino, P., Griswold, M., Hoover, M., Young, R., Day, S., Lloyd Jr., R.M., Reid, C., Morgan, G.F., Winslow, D.L., 2003. J. Clin. Microbiol. 41, 1594-1599.

Lee, P.C., Meisel, D., 1982. J. Phys. Chem. 86, 3391-3395.

Lequin, R.N., 2005. Clin. Chem. 51, 2415-2420.

Li, Y., Wang, Y., Wang, J., Tang, Z., Pounds, J.G., Lin, Y., 2010. Anal. Chem. 82, 7008-7014.

Mao, X., Ma, Y.Q., Zhang, A.G., Zhang, L.R., Zeng, L.W., Liu, G.D., 2009a. Anal. Chem. 81, 1660-1668.

Mao, X., Xu, H., Zeng, Q.X., Zeng, L.W., Liu, G.D., 2009b. Chem. Commun. 3065-3067.

New York Department of Health, 2011. Diagnostic monitoring and resistance laboratory tests for HIV. 〈http://www.hivguidelines.org/wp-content/uploads/di agnostic-monitoring-and-resistance-laboratory-tests-for-hiv-posted-09-262011.pdf). Accessed 12.02.12.

Nie, S.M., Emory, S.R., 1997. Science 275, 1102-1106.

Nkeze, J.N., Constantine, N.T., Zhao, R.Y., 2014. Molecular Diagnostics: 12 Test that changed everything, Molecular and Translational Medicine. In: Highsmith, W.E. (Eds.). Springer Science+Business Media, New York, (Chapter 6).

Rohrman, B.A., Richards-Kortum, R.R., 2012. Lab Chip 12, 3082-3088.

Shen, G.Y., Zhang, S.B., Hu, X., 2013. Clin. Biochem. 46, 1734-1738.

Song, C.Y., Chen, J., Zhao, Y.P., Wang, L.H., 2014. J. Mater. Chem. B 2, 7488-7494.

Sun, Y.H., Kong, R.M., Lu, D.Q., Zhang, X.B., Meng, H.M., Tan, W.H., Shen, G.L., Yu, R. Q., 2011. Chem. Commun. 47, 3840-3842.

Wang, Y., Tang, L.J., Jiang, J.H., 2013a. Anal. Chem. 85, 9213-9220.

Wang, Y.Q. Yan, B., Chen, L.X., 2013b. Chem. Rev. 113, 1391-1428.

Weiss, R.A., 1993. Science 260, 1273-1279.

Wu, Z.T., Liu, Y.Z., Zhou, X.D., Shen, A.G., Hu, J.M., 2013a. Biosens. Bioelectron. 44, $10-15$.

Wu, Z.T., Liu, Y.F., Liu, Y.Z., Xiao, H.M., Shen, A.G., Zhou, X.D., Hu, J.M., 2015b. Biosens, Bioelectron. 65, 375-381.

Xu, H., Mao, X., Zeng, Q.X., Wang, S.F., Kawde, A.N., Liu, G.D., 2009. Anal. Chem. 81, 669-675.

Yoon, J.Y., Choi, N.H., Ko, J.H., Kim, K.H., Lee, S.Y., Choo, J.B., 2013. Biosens. Bioelectron. 47, 62-67.

Zhang, S., Garcia-D'Angeli, A., Brenann, J.P., Huo, O., 2014. Analyst 139, 439-445.

Zhao, P., Wu, Y.Y., Zhu, Y.H., Yang, X.L., Jiang, X., Xiao, J.F., Zhang, Y.X., Li, C.Z., 2014. Nanoscale 6, 3804-3809. 